\title{
Novedades acerca de los trastornos motores del esófago tras la reciente clasificación de Chicago 4.0
}

\author{
Claudia Córdoba, ${ }^{1}$ Agustina Rodil, ${ }^{2}$ Daniel Cisternas ${ }^{3}$ \\ ${ }^{1}$ Sanatorio Allende. Córdoba, Argentina. \\ ${ }^{2}$ Diagnóstico Médico Oroño. Hospital Provincial del Centenario. Rosario, Santa Fe, Argentina. \\ ${ }^{3}$ Clinica Alemana de Santiago, Facultad de Medicina Clinica Alemana-Universidad del Desarrollo. Santiago de Chile, Chile.
}

Acta Gastroenterol Latinoam 2021;51(2):131-142

Recibido: 08/05/2021 / Aceptado: 12/05/2021 / Publicado online: 21/06/2021 / https://doi.org/10.52787/hoho3531

\section{Resumen}

La Clasificación de Chicago reúne los trastornos motores esofágicos diagnosticados por manometría de alta resolución. De los patrones manométricos, algunos son siempre clínicamente relevantes y requieren tratamiento (por ejemplo, la acalasia), mientras que otros pueden ser hallazgos incidentales que no requieren intervención y en los que un manejo agresivo sería contraproducente. Uno de los objetivos de la nueva versión de la Clasificación de Chicago publicada recientemente (CCv4.0) fue distinguir entre trastornos clínicamente relevantes o no. En este sentido, se modificó el protocolo de estudio para incluir degluciones líquidas en posición supino y sentado, estandarizar pruebas de provocación y modificar criterios diagnósticos, incorporando la presencia de sintomas $y$ el soporte de estudios complementarios distintos a la manometría. En esta revisión, desarrollaremos el diagnóstico y tratamiento de la obstrucción de salida de la unión esofagogástrica y los trastornos de hipomotilidad a partir de la CCv4.0.

Correspondencia: Daniel Cisternas

Vitacura 5951, Clínica Alemana de Santiago. Santiago de Chile, Chile Correoelectrónico:dcisternasc@alemana.cl
Palabras claves. Disfagia, manometrría esofágica de alta resolución, clasificación de Chicago, motilidad esofágica ineficiente, contractilidad ausente, obstrucción de salida de la unión gastroesofágica.

\section{Update on Esophageal Motility Disorders after the Recent Chicago Classification 4.0}

\section{Summary}

The Chicago Classification includes esophageal motor disorders diagnosed by high-resolution manometry. Of the manometric patterns, some are always clinically relevant and require treatment (eg, the achalasia), while others may be incidental findings requiring no intervention in which aggressive management would be counterproductive. One of the goals of the new version of the recently published Chicago Classification (CCv4.0) was to distinguish between clinically relevant and non-clinically relevant disorders. With this in mind, the study protocol was modified to include liquid swallows in the supine and sitting positions and provocation tests were standardized. Diagnostic criteria were modified, incorporating the presence of symptoms and the support of complementary studies other than manometry. In this review, we will comment the diagnosis and treatment of esophagogastric junction outlet obstruction and hypomotility disorders based on CCv4.O.

Keywords. Dysphagia, high resolution esophageal manometry, Chicago classification, ineffective esophageal motility, absent contractility, esophagogastric outflow obstruction. 


\section{Abreviaturas}

MAR: Manometría de alta resolución.

CC: Clasificación de Chicago.

CCv4.0: Clasificación de Chicago versión 4.0.

EGJOO: Obstrucción de salida de la unión esofagogástrica.

ERGE: Enfermedad por reflujo gastroesofágico.

DCI: Integral de contracción distal.

CCv3.0: Clasificación de Chicago versión 3.0.

PF: Peristalsis fragmentada.

IEM: Motilidad esofágica inefectiva.

TEA: Tiempo de exposición ácida.

UGE: Unión gastroesofágica.

CA: Contractilidad ausente.

IRP: Presión de relajación integrada.

FLIP: Impedancia planimétrica.

TEB: Estudio baritado temporizado.

\section{Introducción}

La manometría esofágica de alta resolución (MAR) es actualmente el gold standard para el estudio de las enfermedades motoras del esófago. Sobre la base de los hallazgos obtenidos por esta técnica, se desarrolló la Clasificación de Chicago (CC) de trastornos motores esofágicos. Esta clasificación está basada en mecanismos fisiopatológicos y ha permitido estandarizar la nomenclatura utilizando mediciones objetivas. Su uso expandió notablemente la investigación y la aplicabilidad clínica de la MAR. ${ }^{1}$ Recientemente, se publicó su cuarta revisión (CCv4.0). ${ }^{2}$ En ella, se incorporaron modificaciones al protocolo de estudio, que incluyen la realización de degluciones con el paciente en decúbito y en sedestación y el uso de maniobras de provocación para mejorar la sensibilidad y especificidad de los hallazgos (Tabla 1). ${ }^{3}$ Otra modificación relevante consiste en el reconocimiento de que los patrones manométricos pueden no ser específicos, estableciéndose categorías diagnósticas conclusivas y no conclusivas. También, se modificaron los criterios diagnósticos de los trastornos por

Tabla 1. Resumen de las pruebas de provocación sugeridas en el protocolo de CCv4.0

\begin{tabular}{|c|c|c|c|c|c|}
\hline Prueba & Descripción & $\begin{array}{l}\text { Mecanismo } \\
\text { evaluado }\end{array}$ & $\begin{array}{l}\text { Variable } \\
\text { (corte) }\end{array}$ & $\begin{array}{l}\text { Utilidad } \\
\text { escenarios } \\
\text { clínicos }\end{array}$ & Uso en CCv4.0 \\
\hline Agua estándar & $\begin{array}{l}\text { Sentado. } \\
5 \text {-10 } \\
\text { degluciones } \\
\text { de } 5 \mathrm{~mL} \\
\text { cada una. }\end{array}$ & $\begin{array}{l}\text { Relajación } \\
\text { deglutoria de } \\
\text { la UGE. }\end{array}$ & $\operatorname{IRP}(12 \mathrm{mmHg})$. & $\begin{array}{l}\text { Disfagia. } \\
\text { Sospecha de } \\
\text { EGJ00. }\end{array}$ & $\begin{array}{l}\text { Parte de los } \\
\text { criterios } \\
\text { diagnósticos } \\
\text { de EGJ00. }\end{array}$ \\
\hline MRS & $\begin{array}{l}\text { Acostado. } \\
\text { 1-3 sets de } \\
5 \text { degluciones } \\
\text { de } 2 \mathrm{~mL} \\
\text { cada una } \\
\text { (separadas } \\
\text { 2-3 seg). }\end{array}$ & $\begin{array}{l}\text { Reserva } \\
\text { peristáltica. }\end{array}$ & $\mathrm{DCl} \mathrm{MRS} / \mathrm{DCl}(>1)$ & ERGE. & $\begin{array}{l}\text { Criterio de } \\
\text { soporte en } \\
\text { caso de IEM } \\
\text { no conclusiva. }\end{array}$ \\
\hline RDC & $\begin{array}{l}\text { Sentado. } \\
200 \text { mL. } \\
\text { Rápido. Pajilla. }\end{array}$ & $\begin{array}{l}\text { Relajación } \\
\text { deglutoria de } \\
\text { la UGE. }\end{array}$ & $\operatorname{IRP}(12 \mathrm{mmHg})$. & $\begin{array}{l}\text { Disfagia. } \\
\text { Sospecha de } \\
\text { EGJ00. }\end{array}$ & $\begin{array}{l}\text { Criterio de } \\
\text { soporte en } \\
\text { caso de EGJ00 } \\
\text { no conclusiva. }\end{array}$ \\
\hline
\end{tabular}

Pruebas de provocación. MRS: degluciones rápidas múltiples; RDC: desafío de degluciones rápidas; DCl: contractilidad distal integrada; IRP: presión de relajación integrada; EGJOO: obstrucción de salida de la UGE; IEM: motilidad esofágica inefectiva; ERGE: enfermedad por reflujo gastroesofágico. 
hipomotilidad y la obstrucción de salida de la unión esofagogástrica (EGJOO, según sus siglas en inglés), siendo estos los cambios que se abordarán en esta revisión.

\section{Trastornos de hipomotilidad}

\section{Conceptos}

El transporte adecuado del bolo requiere una onda de contracción que tenga un vigor suficiente y, además, una adecuada coordinación temporoespacial. ${ }^{4}$ El vigor de la contracción se mide mediante la integral de contracción distal (DCI, según sus siglas en inglés), que es el producto de la amplitud de la contracción (en $\mathrm{mmHg}$ ) por la extensión (longitud, en $\mathrm{cm}$ ) de esta onda a lo largo del cuerpo esofágico por la duración (tiempo, en seg), y tiene como unidades $\mathrm{mmHg}$ x cm x seg (Figura 1A). La coordinación de la onda se evalúa mediante los defectos peristálticos, que corresponden a la extensión de las zonas del cuerpo esofágico en los que la presión es menor de $20 \mathrm{mmHg}$ (Figura 1B). Roman y cols. determinaron que los defectos peristálticos mayores de $5 \mathrm{~cm}$ siempre se asocian al transporte incompleto de bolo, que aquellos menores de $2 \mathrm{~cm}$ se asocian siempre a transporte completo de bolo, y que los defectos entre 2 y $5 \mathrm{~cm}$ tienen un comportamiento intermedio (Figura 1C). ${ }^{5}$
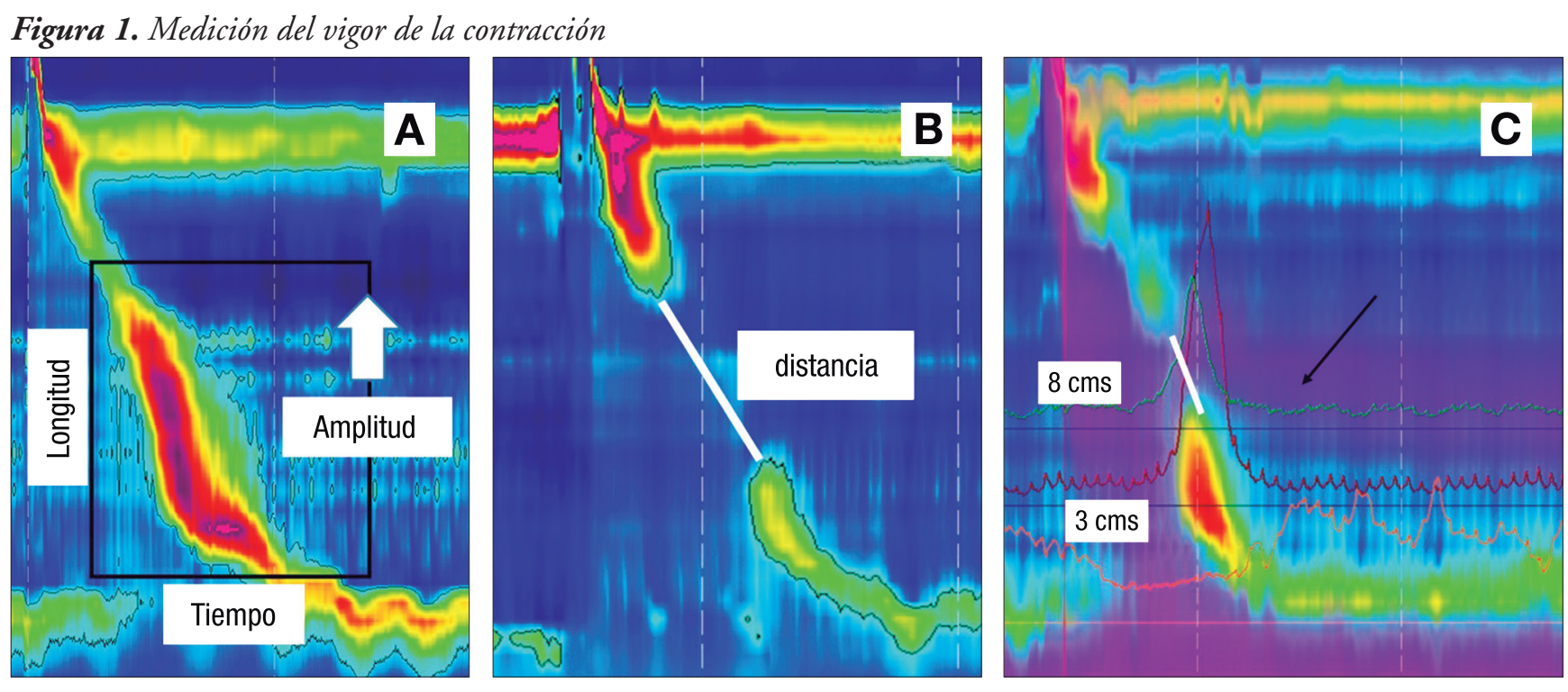

A: Para el cálculo de la integral de contracción distal (DCl) se multiplica el largo de la contracción de musculatura lisa por su duración y amplitud por encima de $20 \mathrm{mmHg}$. B: Para la evaluación de los defectos peristálticos se mide la distancia en el eje longitudinal de las zonas con presión menor a 20 mmHg. C: Manometría de alta resolución con impedanciometría estacionaria: nótese el atrapamiento del bolo evidenciado por impedancia (el tinte púrpura traduce persistencia de contenido luminal en el cuerpo esofágico. Flecha) que ocurre a pesar de la normal amplitud de la contracción del cuerpo esofágico (> $30 \mathrm{mmHg}$ ) medida a 3 y $8 \mathrm{~cm}$ de la unión gastroesofágica, debida a la presencia de un defecto peristáltico (línea blanca).

Conceptualmente, la hipomotilidad esofágica se define manométricamente por degluciones con vigor disminuido y/o presencia de grandes defectos peristálticos, con relajación normal del esfínter esofágico inferior. ${ }^{2,6}$ A lo largo de las diversas versiones de la CC, los criterios para diagnosticar estos dos fenómenos han ido cambiando. Inicialmente, los valores de corte se definieron estadísticamente (usando percentil 95 en poblaciones sanas), pero posteriormente se han utilizado valores de corte para predecir outcomes clínicos. La Tabla 2 compara los criterios diagnósticos de los trastornos de hipomotilidad en CCv3.0 y 4.0. En CCv4.0 el número de trastornos de hipomotilidad se reduce a dos, dado que la peristalsis fragmentada (PF) actualmente forma parte de la motilidad esofágica inefectiva (IEM, según sus siglas en inglés). Esto se debe a que no existen datos que establezcan que se trata de entidades fisiopatológicas, clínica y terapéuticamente diferentes. Además, la PF es una entidad muy infrecuente. ${ }^{3} \mathrm{La}$ PF no fue eliminada pues los defectos peristálticos $>5 \mathrm{~cm}$ demostraron asociación con atrapamiento del bolo deglutido ${ }^{7}$ y con clearance alterado del reflujo, usando el tiempo de exposición ácida (TEA) en monitoreo ambulatorio. ${ }^{8}$

\section{Contractilidad ausente (CA)}

Conceptualmente, corresponde a casos extremos de hipomotilidad, sin ninguna evidencia de actividad motora contráctil medible en el cuerpo esofágico, pero -a diferencia de la acalasia- sin alteraciones de la relajación de la unión gastroesofágica (UGE). Sus criterios diagnósticos nos sufrieron mayores modificaciones en CCv4.0 (Tabla 2). 
Tabla 2. Criterios diagnósticos de los trastornos motores menores según CCv3.0 y CCv4.0

\begin{tabular}{|c|c|c|c|c|}
\hline & \multirow[t]{2}{*}{ CCv3.0 } & \multicolumn{3}{|c|}{ CCv4.0 } \\
\hline & & No conclusiva & Conclusiva & Relevante \\
\hline $\begin{array}{l}\text { Contractilidad } \\
\text { ausente }\end{array}$ & $\begin{array}{l}\mathrm{DCl}<100 \mathrm{mmHg} \\
\text { x cm x seg en el } \\
100 \% \text { de las } \\
\text { degluciones } \\
+ \text { IRP normal. }\end{array}$ & $\begin{array}{l}\mathrm{DCl}<100 \mathrm{mmHg} \text { x cm } \\
\text { x seg en el } 100 \% \text { de } \\
\text { degluciones supino + } \\
\mathrm{IRP} 10-15 \mathrm{mmHg} \\
\text { supino y }<12 \mathrm{mmHg} \\
\text { sedestación. }\end{array}$ & $\begin{array}{l}\mathrm{DCl}<100 \mathrm{mmHg} \text { x cm } \\
\text { x seg en el 100\% de } \\
\text { degluciones acostado + } \\
\text { IRP } 10-15 \text { mmHg supino } \\
\text { y }<12 \mathrm{mmHg} \\
\text { sedestación + TBE y/0 } \\
\text { FLIP que descarten } \\
\text { acalasia. } \\
\text { DCl }<100 \text { mmHg x cm } \\
\text { x seg en el 100\% de } \\
\text { degluciones acostado + } \\
\text { IRP }<10 \text { mmHg supino y } \\
<12 \text { mmHg sedestación. }\end{array}$ & $\begin{array}{l}\text { Siempre } \\
\text { considerada } \\
\text { relevante. }\end{array}$ \\
\hline $\begin{array}{l}\text { Motilidad } \\
\text { esofágica } \\
\text { inefectiva }\end{array}$ & $\begin{array}{l}\mathrm{DCl}<450 \mathrm{mmHg} \\
\mathrm{x} \mathrm{cm} \times \text { seg en } \\
\geq 50 \% \text { de las } \\
\text { degluciones. }\end{array}$ & $\begin{array}{l}50-70 \% \text { degluciones } \\
\text { inefectivas } \\
(\mathrm{DCl}<450 \mathrm{mmHg} x \\
\mathrm{cm} x \text { seg o defecto } \\
\text { peristáltico }>5 \mathrm{~cm} \text { ). }\end{array}$ & $\begin{array}{l}>70 \% \text { deluciones } \\
\text { inefectivas y/0 > 50\% } \\
\text { degluciones fallidas } \\
\text { (DCl < } 100 \mathrm{mmHg} \\
x \mathrm{~cm} \times \text { seg). }\end{array}$ & $\begin{array}{l}\text { Criterios } \\
\text { manométricos + } \\
\text { síntomas } \\
\text { compatibles. }\end{array}$ \\
\hline $\begin{array}{l}\text { Obstrucción de } \\
\text { salida de la } \\
\text { UEG }\end{array}$ & $\begin{array}{l}\text { Mediana de IRP } \\
>15 \mathrm{mmHg}\end{array}$ & $\begin{array}{l}\mathrm{IRP} \geq 15 \mathrm{mmHg} \\
\text { supino + IRP } \\
\geq 12 \mathrm{mmHg} \\
\text { sedestación } \\
+ \text { Presurización } \\
\text { intrabolo } \geq 20 \% .\end{array}$ & $\begin{array}{l}\text { Criterios manométricos } \\
+ \text { TBE y/o FLIP } \\
\text { compatible. }\end{array}$ & $\begin{array}{l}\text { Criterios } \\
\text { manométricos } \\
+ \text { TBE y/o FLIP } \\
\text { compatible + } \\
\text { síntomas } \\
\text { compatibles. }\end{array}$ \\
\hline
\end{tabular}

Comparación de los criterios diagnósticos según CCV3.0 y CCV4.0. Los valores absolutos corresponden al sistema Medtronic. La EGJ00 requiere, además de los criterios de IRP, la presencia de contracciones con cadencia peristáltica.

La CA es un trastorno idiopático, en la mayoría de los casos. En pacientes con esclerodermia, se ha reportado hasta un $40-44 \%$ de CA. ${ }^{9}$ Sin embargo, este hallazgo no debe considerarse sinónimo de esclerodermia ni de los trastornos del colágeno.

La prevalencia reportada de CA, en cohortes de voluntarios sanos, es extremadamente baja $(0,4 \%$ sobre 469 individuos en todo el mundo). ${ }^{10} \mathrm{Su}$ principal consecuencia es la enfermedad por reflujo gastroesofágico (ERGE), habiéndose asociado a síntomas refractarios e injuria mucosa. ${ }^{11}$ Se ha descrito un 3,2\% de CA en pacientes en espera de cirugía antirreflujo, y su presencia se asocia a tiempo de exposición ácida (TEA, por sus siglas en inglés) muy elevados (TEA 17,2\% y 13,5\% en decúbito). ${ }^{11,12}$

Una recomendación muy importante en CCv4.0 es que en el caso de CA con presión de relajación integrada (IRP, según sus siglas en inglés) cercanos al límite superior de la normalidad (entre 10 y $15 \mathrm{mmHg}$ con sistemas Medtronic en supino) y disfagia, debe considerarse el diagnóstico diferencial de acalasia tipo $1 .{ }^{13}$ Esto sucede porque el IRP depende en parte de la presión intrabolo, y esta última puede no estar muy elevada en casos de hipomotildad esofágica muy severa. Para ayudar en la discriminación entre CA y acalasia I en estos casos, la CCv4.0 ha sugerido el uso de la impedancia pla- 
nimétrica (FLIP, según sus siglas en inglés) o el estudio de bario temporizado (TEB, según sus siglas en inglés). La FLIP permite evaluar de manera dirigida la distensibilidad de la unión gastroesofágica. Ponds y colaboradores describieron a un grupo de pacientes con CA y mediana de IRP $<15 \mathrm{mmHg}$ por MAR, pero con disminución de la distensibilidad de la unión esofagogástrica en la FLIP. Estos pacientes fueron tratados como acalasia, con respuesta favorable. ${ }^{14} \mathrm{El} \mathrm{TEB}$ es una prueba más sencilla y ampliamente disponible. Luego de ingerir 200 a $240 \mathrm{ml}$ de bario líquido en posición vertical, la medición de una columna de bario $>5 \mathrm{~cm}$ al minuto $\mathrm{y}>2 \mathrm{~cm}$ a los $5 \mathrm{mi}-$ nutos define un tránsito alterado con una sensibilidad de 85-94\% y una especificidad de 71-86\% para el diagnóstico de acalasia. Combinar bario líquido con la ingesta de una tableta de bario de $13 \mathrm{~mm}$ retenida a los 5 minutos aumenta el rédito diagnóstico al 79,5-100\%. ${ }^{15}$

Adicionalmente, en la MAR con impedanciometría puede estudiarse el atrapamiento del bolo, midiendo por impedancia la columna de agua luego de la ingesta de un bolo de $200 \mathrm{ml}$, con resultados similares al TBE. ${ }^{16}$ Sin embargo, su uso de rutina para diferenciar CA de acalasia I aún no ha sido recomendado en CC.

No existe un tratamiento específico para la CA y no es esperable que se recupere la reserva peristáltica. El manejo consiste en inhibición agresiva de la secreción ácida gástrica, medidas posturales y cambios en el estilo de vida, para reducir el reflujo. En algunos casos, puede ser necesaria una cirugía antirreflujo parcial.

\section{Motilidad esofágica inefectiva (IEM)}

Criterios diagnósticos en CCv4.0. Usando MAR, se ha reportado IEM en entre el 20 y $38 \%$ de los pacientes con síntomas esofágicos. ${ }^{17-19} \mathrm{Si}$ bien la disfagia es el síntoma más frecuente, se presenta solo en el $25 \%$ de los casos. ${ }^{20,21} \mathrm{Sin}$ embargo, con los criterios de CCv3.0, hasta un $15-17 \%$ de sujetos asintomáticos cumplían criterios manométricos de IEM, siendo el desorden más frecuentemente descrito en sanos. ${ }^{22,23}$ Esto sugiere que los criterios de CCv3.0 no son del todo específicos. Así, en CCv4.0, se determinó un criterio más estricto, con un punto de corte de $70 \%$ de degluciones inefectivas para definir IEM (Tabla 2). Este valor de corte discrimina mejor el tránsito anormal del bolo y el reflujo gastroesofágico que el punto de corte $>50 \% .^{8,11,24}$ Por otra parte, se ha demostrado que las degluciones fallidas (DCI menor de $100 \mathrm{mmHg}$ x cm x seg) tienen compromiso más severo que las débiles (DCI entre 100 y $450 \mathrm{mmHg}$ x cm x seg), en términos de transporte incompleto de bolo y TEA. ${ }^{25,26}$
Con esta evidencia, la CCv4.0 define IEM como diagnóstico conclusivo cuando el paciente tiene más de 70\% de degluciones inefectivas (DCI $100-450 \mathrm{mmHg} \mathrm{x} \mathrm{cm}$ $\mathrm{x}$ seg o un defecto peristáltico $>5 \mathrm{~cm}$ ), o al menos $50 \%$ de degluciones fallidas (DCI $<100 \mathrm{mmHg} \times \mathrm{cm}^{2} \mathrm{seg}$ ) ${ }^{5}$, 7, 24,25 (Tabla 2).

La presencia de 50 a 70\% de degluciones inefectivas no es conclusiva para el diagnóstico de IEM. En estos casos, es necesaria evidencia adicional a favor del diagnóstico. ${ }^{8,27}$ Demostrar alteraciones del transporte del bolo mediante impedanciometría o estudio de bario temporizado, y la ausencia de reserva peristáltica en la maniobra de degluciones múltiples, son algunas de las pruebas complementarias que contribuyen al diagnóstico en estos casos. ${ }^{28-31}$

En condiciones normales, tras deglutir rápida y repetidamente, el cuerpo esofágico inhibe su contracción (inhibición deglutoria) y la UGE se relaja. Al salir de este período, ocurre una contracción de mayor amplitud (contracción posdeglutiva) que contribuye al aclaramiento esofágico y cuyo DCI es normalmente mayor al promedio en degluciones aisladas. Cuando esto ocurre, se habla de reserva peristáltica (Figura 2). En CCv4.0, se sugiere evaluarla de manera dirigida, mediante degluciones repetidas múltiples (Tabla 1, Figura 2). ${ }^{32}$ Se sugiere realizar al menos tres series de degluciones rápidas múltiples para evaluar la reserva peristáltica.

En un estudio con 191 pacientes sintomáticos con $50-70 \%$ de degluciones inefectivas y sin reserva peristáltica, se observó que el TEA total y en posición vertical fue significativamente superior que en pacientes con reserva peristáltica, especialmente en posición vertical. ${ }^{11}$ Debe destacarse que la presencia de reserva peristáltica no constituye un criterio diagnóstico de IEM, pero puede aportar información complementaria para apoyar el diagnóstico en casos no concluyentes.

IEM. Relevancia clínica. Si bien existen algunos reportes de evolución a acalasia, se trata de casos aislados. ${ }^{33,} 34$ Se considera que la IEM tiene un curso benigno. Ravi y cols. no lograron demostrar diferencias en la evolución clínica a seis años de pacientes sintomáticos con MAR normal versus pacientes con MAR con hipomotilidad. ${ }^{35}$

La IEM se ha descrito asociada a múltiples condiciones (Tabla 3). Desde un punto de vista fisiopatológico, es un trastorno muy heterogéneo, habiéndose descrito alteraciones neuropáticas aferentes y eferentes, así como alteraciones miopáticas y fibrosis. ${ }^{26,36,37}$ La preponderancia de cada uno de estos mecanismos parece depender fundamentalmente de la etiología de la IEM. 
Figura 2. Degluciones rápidas múltiples y reserva peristáltica
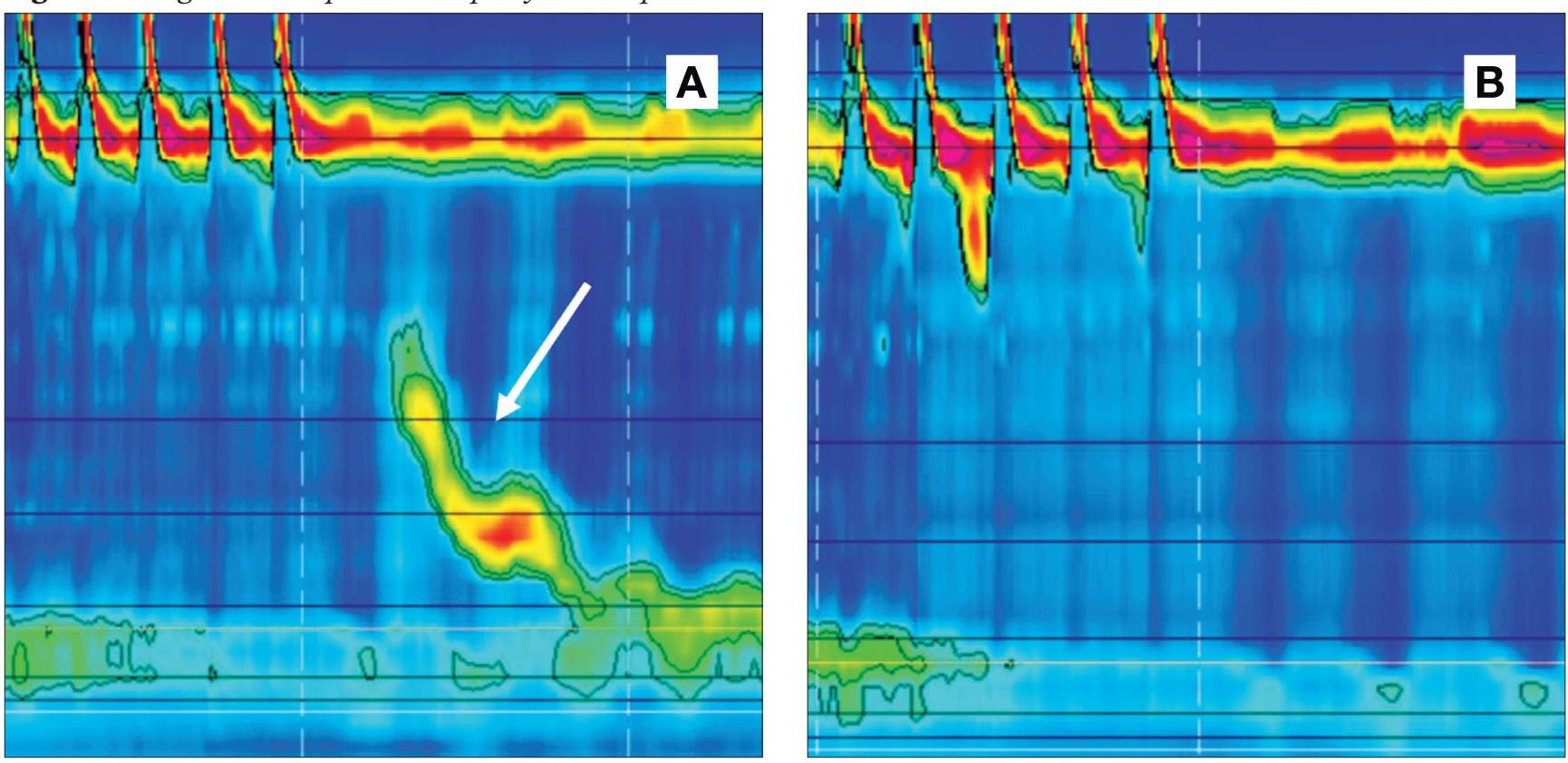

Degluciones rápidas múltiples para la evaluación de la reserva peristáltica. En A se evidencia una adecuada contracción posdeglutiva, mientras que en B no la hay.

Tabla 3. Algunas condiciones asociadas a IEM"3-75 y a la EGJOO 58, 59, 76-78

\begin{tabular}{ll}
\hline IEM & EGJ00 \\
\hline Esclerodermia & Variantes o formas iniciales de acalasia \\
Enfermedades del mesénquima & Enfermedad de Chagas \\
Enfermedad por reflujo gastroesofágico & Pseudoacalasia \\
Diabetes mellitus con disfunción autonómica & Obstrucciones mecánicas de la UGE \\
Amiloidosis & $\bullet$ Hernia hiatal \\
Consumo excesivo de alcohol & $\bullet$ Cáncer de la UGE \\
Esofagitis eosinofilica & $\bullet$ Posfundoplicatura \\
Enfermedad de Parkinson & $\bullet$ Estenosis péptica \\
& $\bullet$ Anillo de Schatski obstructivo \\
& $\bullet$ Compresión extrínseca (adenopatías, vasculares) \\
& Esofagitis eosinofilica \\
& Opioides \\
& Artefactual \\
\hline
\end{tabular}

Ante el hallazgo de IEM, el contexto clínico del paciente determina la relevancia del diagnóstico. Los principales escenarios de relevancia clínica son la disfagia y la enfermedad por reflujo gastroesofágico.

IEM y enfermedad por reflujo gastroesofágico (ERGE). La hipomotilidad disminuye el aclaramiento esofágico, lo que representa un mecanismo fisiopatológico en la ERGE. ${ }^{38}$ Pese a lo frecuente de su asociación, la IEM no es patognomónica de ERGE y más del $50 \%$ de los pacientes con ERGE tienen motilidad esofágica normal. ${ }^{39}$ Sin embargo, en pacientes con ERGE asociado a IEM se ha descrito TAE más prolongado ${ }^{8,27}$ y una mayor frecuencia de lesiones endos- 
cópicas, como el esófago de Barrett, ${ }^{38,40}$ que en sujetos con motilidad normal.

En un estudio multicéntrico de 351 pacientes, se evidenció que $\geq 50 \%$ degluciones fallidas, $\geq 70 \%$ degluciones inefectivas así como $\geq 70 \%$ degluciones con defectos peristálticos grandes predicen independientemente TAE anormal. ${ }^{8}$

IEM y disfagia. A pesar de haber sido reiteradamente estudiado, no se ha logrado determinar una adecuada correlación entre hipomotilidad y síntomas de disfagia. ${ }^{41,42}$ Lazarescu y col. no pudieron demostrar la correlación entre percepción de disfagia e hipomotilidad inducida por sildenafil, en un grupo de individuos sanos. ${ }^{43}$ Cisternas y col. no lograron demostrar la correlación entre el DCI y la percepción del paso de bolo en individuos sanos. ${ }^{44}$ Sin embargo, sí demostraron una correlación significativa entre percepción del paso de bolo y el nivel de ansiedad $\left(\mathrm{R}^{2}=0,7\right)$ en el subgrupo de individuos con hipomotilidad, sugiriendo que, en el contexto de un fenómeno frecuente, como la hipomotilidad y el transporte incompleto de bolo, la ansiedad aumenta la probabilidad de percepción, tal vez mediante hipersensibilidad visceral.

IEM y disfagia posfundoplicatura. Se ha descrito que entre el 5 y $10 \%$ de los pacientes presentan disfagia prolongada (más de seis meses), luego de una cirugía antirreflujo. ${ }^{45}, 46 \mathrm{Al}$ aumentar la presión de la unión gastroesofágica después de la cirugía, se ha considerado que la presencia de hipomotilidad esofágica precirugía sería un predictor de disfagia posquirúrgica. Sin embargo, la evaluación del vigor mediante DCI no ha logrado ser un predictor importante de la disfagia poscirugía: Kapadia y col. demostraron solo una pequeña correlación entre el DCI y el riesgo de disfagia posquirúrgica $(\mathrm{r}=-0,29) \cdot{ }^{47}$ Siegal y col. probaron que el DCI solo se asocia a disfagia posquirúrgica en los pacientes que presentaban disfagia antes de la cirugía. ${ }^{48}$ Sin embargo, la evaluación de la reserva peristáltica parece ser más promisoria para una adecuada predicción de la disfagia poscirugía. ${ }^{49}, 50$

IEM. Tratamiento. El manejo de los trastornos por hipomotilidad, en pacientes sintomáticos, es complejo porque no existe un tratamiento específico que restaure la motilidad o mejore los síntomas. ${ }^{51}$

Los pacientes con trastornos por hipomotilidad en MAR, asintomáticos y sin evidencia de ERGE, no re- quieren tratamiento. En los pacientes con evidencia de ERGE, se realizará tratamiento del reflujo: cambios en la dieta y en el estilo de vida, terapia antisecretora, $y$, en algunos casos, puede ser necesario realizar cirugía antirreflujo. ${ }^{38,52}$

Los agentes procinéticos convencionales por vía oral (metoclopramida, domperidona) no han demostrado eficacia en la hipomotilidad esofágica ni mejoría de los síntomas. ${ }^{3,26}$ Tampoco hay evidencia de un aumento del vigor contráctil ni en el control de síntomas con agonistas 5-HT4 como Mosapride y Reverexepride. ${ }^{53-55}$ Respecto del Prucalopride, hay evidencia muy limitada de mejoría del TEA y de los síntomas esofágicos en cuatro pacientes con ERGE en tratamiento por estreñimiento concomitante. ${ }^{56}$

\section{Obstrucción de salida de la unión esofagogás- trica (EGJOO)}

La relajación deglutoria de la UGE en MAR se mide mediante la presión de relajación integrada (IRP, según sus siglas en inglés), que tiene un valor máximo normal de $15 \mathrm{mmHg}$ en posición supina en los sistemas Medtronic (Figura 3). La obstrucción del flujo de salida de la UGE (EGJOO, según sus siglas en inglés) corresponde a un patrón manométrico caracterizado por insuficiente relajación de la UGE durante la deglución, pero -a diferencia de la acalasia- existen evidencias de contracciones del cuerpo esofágico con cadencia peristáltica. Se presenta en un grupo muy heterogéneo de condiciones, incluidas las formas iniciales o variantes de acalasia, obstrucciones mecánicas, esofagitis eosinofílica, uso de fármacos como opioides, entre otros (Tabla 3). ${ }^{57,58}$ Por lo tanto, estos pacientes siempre deben ser evaluados de forma exhaustiva, incluso endoscopía con biopsias de mucosa y estudios por imágenes.

Esta heterogeneidad también se refleja en el impacto clínico de este hallazgo. Por una parte, se ha descrito progresión a acalasia en el 2-4\% de los casos. ${ }^{57-59}$ Por otro lado, este patrón se ha descrito en 6-7\% de individuos sanos asintomáticos, usando los criterios diagnósticos de CCv3.0. ${ }^{60}$ Incluso en individuos sintomáticos, en estudios de historia natural, se muestran que, en un alto porcentaje de los casos, los síntomas remiten de forma espontánea a lo largo del tiempo..$^{58,61}$

El grupo de trabajo de CCv4.0 se propuso como objetivo primordial aumentar la especificidad de los criterios de EGJOO, de modo de identificar mejor a los pacientes con pronóstico adverso y evitar tratamientos invasivos en pacientes con buen pronóstico. 
Figura 3. Medición de la relajación deglutiva de la UGE
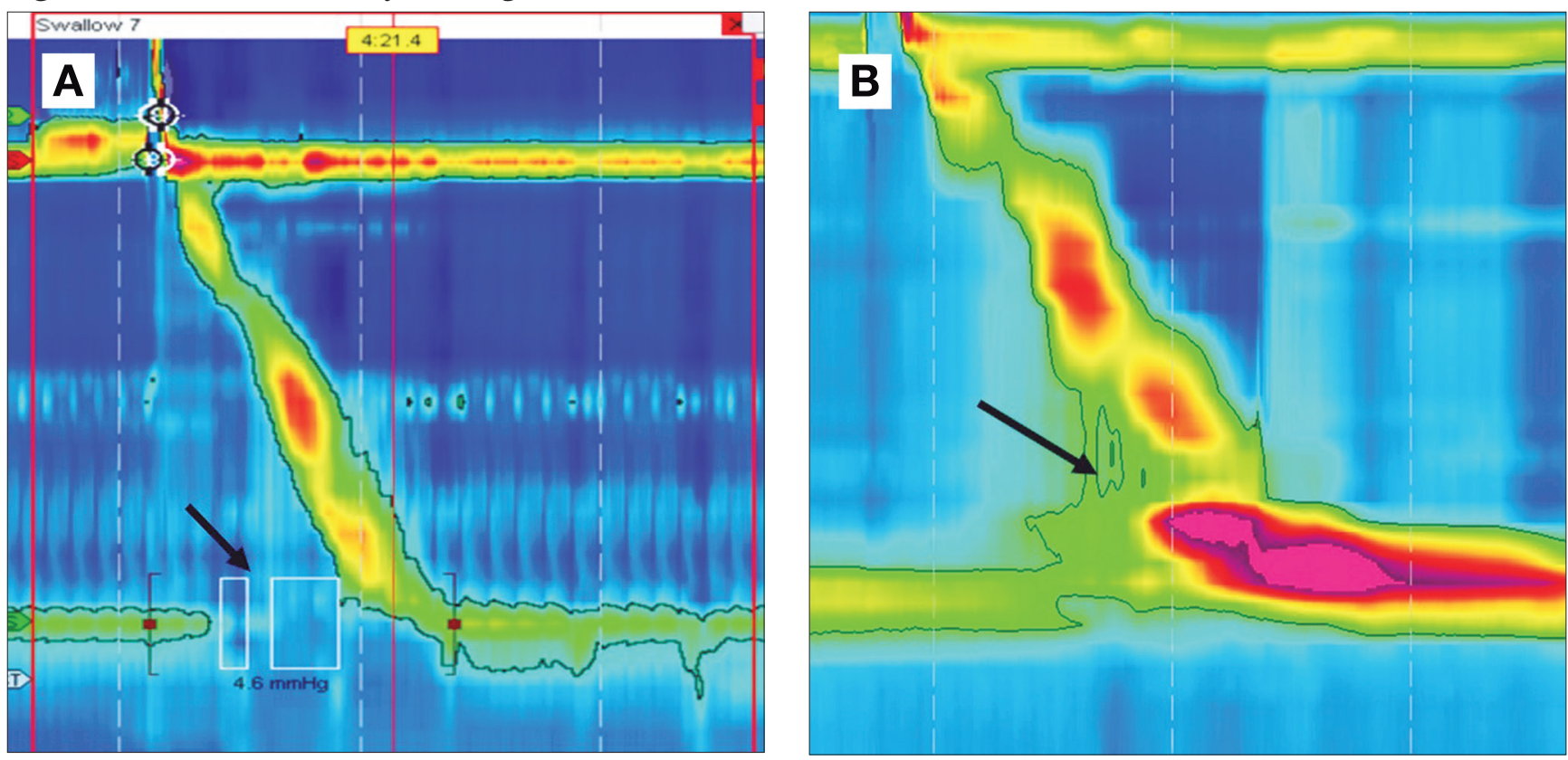

Evaluación de la relajación de la UGE mediante la presión de relajación integral (IRP. A. Flecha). B: Usando la isobara en $30 \mathrm{mmHg}$ se evidencia que el bolo aumenta su presión ante la dificultad del paso a través de la UGE (flecha), fenómeno manométrico llamado presurización compartimentalizada.

En primer lugar, existe evidencia de que la medición de presión deglutoria en la UGE puede estar sobrevalorada artificialmente, probablemente por doblez de la sonda al apoyarse en la curvatura mayor del estómago. ${ }^{62}$ Para minimizar este efecto, se ha propuesto un cambio de posición del paciente, con la idea de que al sentarse la sonda pudiese movilizarse. Krause y col. mostraron que ninguno de los pacientes con criterios de CCv3.0 de EGJOO, que normalizaban el IRP al sentarse, (IRP $<12 \mathrm{mmHg}$ ) tenía evidencias de obstrucción de la UGE demostrable en un TEB. ${ }^{63}$ Por otra parte, es esperable que, en una obstrucción real de la UGE, el bolo aumente su presión al encontrar dificultad de paso. Ong y col. evaluaron a 122 pacientes con criterios de EGJOO según CCv3.0. De ellos, 87 pacientes no presentaban evidencias de presurización del bolo en ninguna deglución (llamada presurización compartimentalizada [Figura 3]). Ninguno de ellos presentó una evolución adversa ni requirió tratamiento específico en el mediano plazo. ${ }^{61}$ Con esta información, la CCv4.0 ha agregado a los criterios manométricos de EGJOO la exigencia de un IRP elevado tanto en posición supina como en sedestación, además de la exigencia de presurización compartimentalizada (Tabla 2).

Debe consignarse que las evidencias de obstrucción en pruebas complementarias como el desafío de degluciones rápidas con $200 \mathrm{~mL}$, el uso de comida sólida o en pro- vocación farmacológica (con nitrito de amilo o colecistoquinia) tienen sensibilidades y especificidades menores que las degluciones sentadas con agua para el diagnóstico conclusivo de EGJOO. ${ }^{63}$ Por esto, permiten aumentar la confianza en un diagnóstico conclusivo cuando están presentes, pero por sí solas no son capaces de establecer el diagnóstico de EGJOO. ${ }^{2}$

Con la idea de mejorar aún más la especificidad del diagnóstico de EGJOO, se sugiere la adición de evidencias de obstrucción con métodos más allá de la manometría, tales como el estudio baritado temporizado (TEB, según sus siglas en inglés) y la FLIP. Con esto, CCv4.0 sugiere que, cuando se cumplen los criterios manométricos descritos, se establezca un diagnóstico no conclusivo de EGJOO. Si a posteriori se agregan evidencias de obstrucción en el TBE o en la FLIP, debe establecerse el diagnóstico conclusivo de EGJOO (Tabla 2). ${ }^{2}$

En segundo lugar, se ha descrito, en varias series, que los pacientes con criterios de EGJOO que no presentan disfagia al momento de la MAR tienen un excelente pronóstico en el seguimiento. ${ }^{61}$ Con esto, CCv4.0 sugiere exigir la presencia de síntomas para considerar una EGJOO conclusiva como accionable (que amerita tratamiento específico). ${ }^{2}$

\section{EGJOO. Abordaje}

Como en todos los pacientes sometidos a una MAR, 
es recomendable tener previamente un estudio endoscópico, incluidas biopsias del esófago, en caso de síntomas como disfagia o dolor torácico. ${ }^{64} \mathrm{Al}$ momento de la MAR, es importante contar con una adecuada evaluación de la historia, especialmente para tener antecedentes del uso de fármacos como opioides y de cirugías gastroesofágicas, además de la evaluación de los síntomas.

Por lo mencionado anteriormente, los pacientes sin disfagia ni dolor torácico, así como los que tienen algunas alteraciones pero no alcanzan todos los criterios manométricos de CCv4.0 para EGJOO no conclusivo, deben ser manejados de manera conservadora, con la recomendación de hacer controles periódicos o en caso de que aparezcan síntomas. ${ }^{64,65}$

Los pacientes sintomáticos con criterios manométricos de una EGJOO no conclusiva deben ser sometidos a TBE o FLIP para determinar si la obstrucción es real, y el diagnóstico puede ser considerado conclusivo. ${ }^{2}$

En los pacientes con diagnóstico conclusivo, debe hacerse una evaluación de las posibles etiologías, que incluya idealmente una imagen de la zona (una TAC de tórax, habitualmente) y una exhaustiva revisión de la imagen endoscópica para descartar la presencia de una lesión neoplásica a nivel de la UGE. En pacientes de edad avanzada, especialmente con rápida pérdida de peso, debe estudiarse la presencia de neoplasias (especialmente pulmonares y ginecológicas) que pueden manifestar una alteración esofágica paraneoplásica. ${ }^{66,67} \mathrm{Si}$ bien aún no existen datos para validar esta conducta, dado que la enfermedad de Chagas puede presentar diversos patrones manométricos similares a la acalasia, en muchos centros de América del Sur se solicita serología de Chagas a estos pacientes.

\section{EGJOO conclusiva relevante. Tratamiento}

Debe enfatizarse el manejo cauteloso de estos pacientes, reservando las terapias invasivas para casos de diagnóstico conclusivo con síntomas severos. La observación clínica de cuadros moderados es recomendable. ${ }^{68}$

Dado que la obstrucción de salida de la unión esofagogástrica reúne cuadros clínicos heterogéneos, el tratamiento se apoya en el diagnóstico preciso de la etiología subyacente.

Por ejemplo, se ha descrito que la mayoría de los pacientes con disfagia, esofagitis eosinofílica y patrón manométrico de EGJOO y/o acalasia responden favorablemente al uso de inhibidores de bomba de protones o inmunosupresores, sin requerir terapias descompresivas. ${ }^{69}$ Por otro lado, las obstrucciones mecánicas, como una hernia paresofágica obstructiva, requieren tratamiento dirigido ya que no responden bien a fármacos. ${ }^{57}$
En los casos sin una etiología específica tratable, los tratamientos tendientes a descomprimir la UGE son los indicados. En este contexto, el uso de fármacos, como los antagonistas de calcio, suele ser ineficaz. ${ }^{70}$ Los pacientes con síntomas relevantes persistentes en el tiempo, que cumplen los resultados de MAR y estudios complementarios mencionados, corresponden a un subgrupo pequeño altamente seleccionado que presenta tasas de respuesta a la descompresión de la UGE similares a los pacientes con acalasia. ${ }^{71}$ Entre las opciones de descompresión, el uso de Botox y POEM ha mostrado tasas de efectividad entre el 75 y $95 \%$ aproximadamente. ${ }^{58,71,72}$ La decisión del tipo de terapia descompresiva dependerá de la experiencia del centro y de las características clínicas del paciente, considerando que la terapia con Botox solo dura algunos meses.

Conflictos de interés. Ninguno de los autores tiene conflictos de interés en relación con este manuscrito.

Financiamiento. Este manuscrito no contó con ninguna forma de financiamiento.

\section{Aviso de derechos de autor}

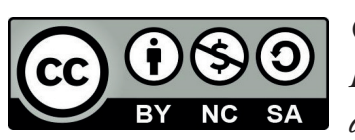

(C) 2021 Acta Gastroenterológica Latinoamericana. Este es un artículo de acceso abierto publicado bajo los términos de la Licencia Creative Commons Attribution (CC BY-NC-SA 4.0), la cual permite el uso, la distribución $y$ la reproducción de forma no comercial, siempre que se cite al autor y la fuente original.

Cite este artículo como: Córdoba C, Rodil A, Cisternas D. Novedades acerca de los trastornos motores del esófago tras la reciente clasificación de Chicago 4.0. Acta Gastroenterol Latinoam. 2021;51(2):131-42. https://doi.org/10.52787/ hoho3531

\section{Referencias}

1. Yadlapati RPJ, Fox MR, Bredenoord AJ, Kahrilas PJ. What is new in Chicago Classification version 4.0? Neurogastroenterol Motil. 2021;33(1):e14053.

2. Yadlapati R, Kahrilas PJ, Fox MR, Bredenoord AJ, Prakash Gyawali C, Roman S, Babaei A, Mittal RK, Rommel N, Savarino E, Sifrim D, Smout A, Vaezi MF, Zerbib F, Akiyama J, Bhatia S, Bor S, Carlson DA, Chen JW, Cisternas D, Cock C, Coss-Adame E, de Bortoli N, Defilippi C, Fass R, Ghoshal UC, Gonlachanvit S, Hani A, Hebbard GS, Wook Jung K, Katz P, Katzka DA, Khan A, Kohn GP, Lazarescu A, Lengliner J, Mittal SK, Omari T, Park MI, Penagini R, Pohl D, Richter JE, Serra J, Sweis R, Tack J, Tatum RP, Tutuian R, Vela MF, Wong RK, Wu JC, Xiao Y, Pandolfino JE. Esophageal motility disorders on high-resolution manometry: Chicago classification version $4.0((\mathrm{c}))$. Neurogastroenterol Motil. 2021;33:e14058. 
3. Gyawali CP, Zerbib F, Bhatia S, Cisternas D, Coss-Adame E, Lazarescu A, Pohl D, Yadlapati R, Penagini R, Pandolfino J. Chicago Classification update (V4.0): Technical review on diagnostic criteria for ineffective esophageal motility and absent contractility. Neurogastroenterol Motil. 2021:e14134.

4. Ghosh SK, Janiak P, Schwizer W, Hebbard GS, Brasseur JG. Physiology of the esophageal pressure transition zone: separate contraction waves above and below. Am J Physiol Gastrointest Liver Physiol. 2006;290:G568-76.

5. Roman S, Lin Z, Kwiatek MA, Pandolfino JE, Kahrilas PJ. Weak peristalsis in esophageal pressure topography: classification and association with Dysphagia. Am J Gastroenterol 2011;106:349-56.

6. Kahrilas PJ, Bredenoord AJ, Fox M, Gyawali CP, Roman S, Smout AJ, Pandolfino JE. The Chicago Classification of esophageal motility disorders, v3.0. Neurogastroenterol Motil 2015;27:160-74.

7. Bulsiewicz WJ, Kahrilas PJ, Kwiatek MA, Ghosh SK, Meek A, Pandolfino JE. Esophageal pressure topography criteria indicative of incomplete bolus clearance: a study using high-resolution impedance manometry. Am J Gastroenterol. 2009;104:2721-8.

8. Rogers BD, Rengarajan A, Mauro A, Ghisa M, De Bortoli N, Cicala M, Ribolsi M, Penagini R, Savarino E, Gyawali CP. Fragmented and failed swallows on esophageal high-resolution manometry associate with abnormal reflux burden better than weak swallows. Neurogastroenterol Motil 2020;32:e13736.

9. Carlson DACM, Kimmel JN, Patel A, Gyawali CP, Hinchcliff M, Griffing WL, Pandolfino JE, Vela MF. Loss of Peristaltic Reserve, Determined by Multiple Rapid Swallows, Is the Most Frequent Esophageal Motility Abnormality in Patients With Systemic Sclerosis. Clin Gastroenterol Hepatol. 2016;14(10):1502-6.

10. Rengarajan ARB, Wong Z, Tolone S, Sifrim D, Serra J, Savarino E, Roman S, Remes-Troche JM, Ramos R, Pérez de la Serna J, Pauwels A, Leguizamo AM, Lee YY, Kawamura O, Hayat J, Hani A, Gonlachanvit S, Cisternas D, Carlson D, Bor S, Bhatia S, Abrahao L, Pandolfino J, Gyawali CP. High-Resolution Manometry Thresholds and Motor Patterns Among Asymptomatic Individuals. Clin Gastroenterol Hepatol. 2020;20:1542-3565.

11. Quader F, Rogers B, Sievers T, Mumtaz S, Lee M, Lu T, Gyawali CP. Contraction Reserve With Ineffective Esophageal Motility on Esophageal High-Resolution Manometry is Associated With Lower Acid Exposure Times Compared With Absent Contraction Reserve. Am J Gastroenterol. 2020;115:1981-8.

12. Chan WW, Haroian LR, Gyawali CP. Value of preoperative esophageal function studies before laparoscopic antireflux surgery. Surg Endosc. 2011;25:2943-9.

13. Lin Z, Kahrilas PJ, Roman S, Boris L, Carlson D, Pandolfino JE. Refining the criterion for an abnormal Integrated Relaxation Pressure in esophageal pressure topography based on the pattern of esophageal contractility using a classification and regression tree model. Neurogastroenterol Motil. 2012;24:e356-63.

14. Ponds FA, Bredenoord AJ, Kessing BF, Smout AJ. Esophagogastric junction distensibility identifies achalasia subgroup with manometrically normal esophagogastric junction relaxation. Neurogastroenterol Motil. 2017;29.

15. Blonski W, Kumar A, Feldman J, Richter JE. Timed Barium Swallow: Diagnostic Role and Predictive Value in Untreated Achalasia, Esophagogastric Junction Outflow Obstruction, and Non-Achalasia Dysphagia. Am J Gastroenterol. 2018;113:196-203.
16. Cho YK, Lipowska AM, Nicodème F, Teitelbaum EN, Hungness ES, Johnston ER, Gawron A, Kahrilas PJ, Pandolfino JE. Assessing bolus retention in achalasia using high-resolution manometry with impedance: a comparator study with timed barium esophagram. Am J Gastroenterol. 2014;109:829-35.

17. Boland K, Abdul-Hussein M, Tutuian R, Castell DO. Characteristics of Consecutive Esophageal Motility Diagnoses After a Decade of Change. J Clin Gastroenterol. 2016;50:301-6.

18. Liu Z, Liao J, Tian D, Liu M, Dan Z, Yu Q. Assessment of Esophageal High-Resolution Impedance Manometry in Patients with Nonobstructive Dysphagia. Gastroenterol Res Pract. 2018;2018:6272515.

19. Wang D, Wang X, Yu Y, Xu X, Wang J, Jia Y, Xu H. Assessment of Esophageal Motor Disorders Using High-resolution Manometry in Esophageal Dysphagia With Normal Endoscopy. J Neurogastroenterol Motil. 2019;25:61-7.

20. Blonski W, Vela M, Safder A, Hila A, Castell DO. Revised criterion for diagnosis of ineffective esophageal motility is associated with more frequent dysphagia and greater bolus transit abnormalities. Am J Gastroenterol. 2008;103:699-704.

21. Abdel Jalil AA, Castell DO. Ineffective Esophageal Motility (IEM): the Old-New Frontier in Esophagology. Curr Gastroenterol Rep. 2016;18:1.

22. Rengarajan A, Rogers BD, Wong Z, Tolone S, Sifrim D, Serra J, Savarino E, Roman S, Remes-Troche JM, Ramos R, Pérez de la Serna J, Pauwels A, Leguizamo AM, Lee YY, Kawamura O, Hayat J, Hani A, Gonlachanvit S, Cisternas D, Carlson D, Bor S, Bhatia S, Abrahao L, Pandolfino J, Gyawali CP. High-Resolution Manometry Thresholds and Motor Patterns Among Asymptomatic Individuals. Clin Gastroenterol Hepatol. 2020.

23. Hollenstein M, Thwaites P, Bütikofer S, Heinrich H, Sauter M, Ulmer I, Pohl D, Ang D, Eberli D, Schwizer W, Fried M, Distler O, Fox M, Misselwitz B. Pharyngeal swallowing and oesophageal motility during a solid meal test: a prospective study in healthy volunteers and patients with major motility disorders. Lancet Gastroenterol Hepatol. 2017;2:644-53.

24. Zerbib F, Marín I, Cisternas D, Abrahao L Jr, Hani A, Leguizamo AM, Remes-Troche JM, Pérez de la Serna J, Ruiz de Leon A, Serra J. Ineffective esophageal motility and bolus clearance. A study with combined high-resolution manometry and impedance in asymptomatic controls and patients. Neurogastroenterol Motil. 2020;32:e13876.

25. Jain A, Baker JR, Chen JW. In ineffective esophageal motility, failed swallows are more functionally relevant than weak swallows. Neurogastroenterol Motil. 2018;30:e13297.

26. Gyawali CP, Sifrim D, Carlson DA, Hawn M, Katzka DA, Pandolfino JE, Penagini R, Roman S, Savarino E, Tatum R, Vaezi M, Clarke JO, Triadafilopoulos G. Ineffective esophageal motility: Concepts, future directions, and conclusions from the Stanford 2018 symposium. Neurogastroenterol Motil. 2019:e13584.

27. Rengarajan A, Bolkhir A, Gor P, Wang D, Munigala S, Gyawali CP. Esophagogastric junction and esophageal body contraction metrics on high-resolution manometry predict esophageal acid burden. Neurogastroenterol Motil. 2018;30:e13267.

28. Chugh P, Collazo T, Dworkin B, Jodorkovsky D. Ineffective Esophageal Motility Is Associated with Impaired Bolus Clearance but Does Not Correlate with Severity of Dysphagia. Dig Dis Sci. 2019;64:811-4. 
29. Lin Z, Nicodeme F, Lin CY, Mogni B, Friesen L, Kahrilas PJ, Pandolfino JE. Parameters for quantifying bolus retention with high-resolution impedance manometry. Neurogastroenterol Motil. 2014;26:929-36.

30. Wu JF, Tsai IJ, Tong TW, Lin YC, Yang CH, Tseng PH. Pressure-impedance analysis: Assist the diagnosis and classification of ineffective esophageal motility disorder. J Gastroenterol Hepatol. 2020;35:1317-24.

31. Martinucci I, Savarino EV, Pandolfino JE, Russo S, Bellini M, Tolone S, Tutuian R, Roman S, Furnari M, Frazzoni M, Macchia L, Savarino V, Marchi S, de Bortoli N. Vigor of peristalsis during multiple rapid swallows is inversely correlated with acid exposure time in patients with NERD. Neurogastroenterol Motil. 2016;28:243-50.

32. Price LH, Li Y, Patel A, Gyawali CP. Reproducibility patterns of multiple rapid swallows during high resolution esophageal manometry provide insights into esophageal pathophysiology. Neurogastroenterol Motil. 2014;26:646-53.

33. Samo S, Carlson DA, Kahrilas PJ, Pandolfino JE. Ineffective Esophageal Motility Progressing into Distal Esophageal Spasm and Then Type III Achalasia. ACG Case Rep J. 2016;3:e183.

34. Naftali T, Levit T, Pomeranz I, Benjaminov FS, Konikoff FM. Nonspecific esophageal motility disorders may be an early stage of a specific disorder, particularly achalasia. Dis Esophagus. 2009;22:611-5.

35. Ravi K, Friesen L, Issaka R, Kahrilas PJ, Pandolfino JE. Long-term Outcomes of Patients With Normal or Minor Motor Function Abnormalities Detected by High-resolution Esophageal Manometry. Clin Gastroenterol Hepatol. 2015;13:1416-23.

36. Kim HS, Park H, Lim JH, Choi SH, Park C, Lee SI, Conklin JL. Morphometric evaluation of oesophageal wall in patients with nutcracker oesophagus and ineffective oesophageal motility. Neurogastroenterol Motil. 2008;20:869-76.

37. Chen JH. Ineffective esophageal motility and the vagus: current challenges and future prospects. Clin Exp Gastroenterol. 2016;9:291-9.

38. Gyawali CP, Kahrilas PJ, Savarino E, Zerbib F, Mion F, Smout A, Vaezi M, Sifrim D, Fox MR, Vela MF, Tutuian R, Tack J, Bredenoord AJ, Pandolfino J, Roman S. Modern diagnosis of GERD: the Lyon Consensus. Gut. 2018;67:1351-62.

39. Diener U, Patti MG, Molena D, Fisichella PM, Way LW. Esophageal dysmotility and gastroesophageal reflux disease. J Gastrointest Surg. 2001;5:260-5.

40. Ang D, Blondeau K, Sifrim D, Tack J. The spectrum of motor function abnormalities in gastroesophageal reflux disease and Barrett's esophagus. Digestion. 2009;79:158-68.

41. Chen CL, Yi CH. Clinical correlates of dysphagia to oesophageal dysmotility: studies using combined manometry and impedance. Neurogastroenterol Motil. 2008;20:611-7.

42. Xiao Y, Kahrilas PJ, Nicodeme F, Lin Z, Roman S, Pandolfino JE. Lack of correlation between HRM metrics and symptoms during the manometric protocol. Am J Gastroenterol. 2014;109:521-6.

43. Lazarescu A, Karamanolis G, Aprile L, De Oliveira RB, Dantas R, Sifrim D. Perception of dysphagia: lack of correlation with objective measurements of esophageal function. Neurogastroenterol Motil. 2010;22:1292-7, e336-7.
44. Cisternas D, Scheerens C, Omari T, Monrroy H, Hani A, Leguizamo A, Bilder C, Ditaranto A, Ruiz de Leon A, Pérez de la Serna J, Valdovinos MA, Coello R, Abrahao L, Remes-Troche J, Meixueiro A, Zavala MA, Marín I, Serra J. Anxiety can significantly explain bolus perception in the context of hypotensive esophageal motility: Results of a large multicenter study in asymptomatic individuals. Neurogastroenterol Motil. 2017;29.

45. Bammer T, Hinder RA, Klaus A, Klingler PJ. Five to eight-year outcome of the first laparoscopic Nissen fundoplications. J Gastrointest Surg. 2001;5:42-8.

46. Dallemagne B, Weerts J, Markiewicz S, Dewandre JM, Wahlen C, Monami B, Jehaes C. Clinical results of laparoscopic fundoplication at ten years after surgery. Surg Endosc. 2006;20:159-65.

47. Kapadia S, Osler T, Lee A, Borrazzo E. The role of preoperative high resolution manometry in predicting dysphagia after laparoscopic Nissen fundoplication. Surg Endosc. 2018;32:2365-72.

48. Siegal SR, Dunst CM, Robinson B, Dewey EN, Swanstrom LL, DeMeester SR. Preoperative High-Resolution Manometry Criteria are Associated with Dysphagia After Nissen Fundoplication. World J Surg. 2019;43:1062-7.

49. Shaker A, Stoikes N, Drapekin J, Kushnir V, Brunt LM, Gyawali CP. Multiple rapid swallow responses during esophageal high-resolution manometry reflect esophageal body peristaltic reserve. Am J Gastroenterol. 2013;108:1706-12.

50. Stoikes N, Drapekin J, Kushnir V, Shaker A, Brunt LM, Gyawali CP. The value of multiple rapid swallows during preoperative esophageal manometry before laparoscopic antireflux surgery. Surg Endosc. 2012;26:3401-7.

51. Smout A, Fox M. Weak and absent peristalsis. Neurogastroenterol Motil. 2012;24(Suppl 1):40-7.

52. Triadafilopoulos GTA, Shetler KP, Clarke J. Clinical and $\mathrm{pH}$ study characteristics in reflux patients with and without ineffective oesophageal motility (IEM). BMJ Open Gastroenterol. 2016;3(1):e000126.

53. Liu Q, Feng CC, Wang EM, Yan XJ, Chen SL. Efficacy of mosapride plus proton pump inhibitors for treatment of gastroesophageal reflux disease: a systematic review. World J Gastroenterol. 2013;19:9111-8.

54. Shaheen NJ, Adler J, Dedrie S, Johnson D, Malfertheiner P, Miner P, Meulemans A, Poole L, Tack J, Thielemans L, Troy S, Vakil N, Zerbib F, Ruth M. Randomised clinical trial: the 5-HT4 agonist revexepride in patients with gastro-esophageal reflux disease who have persistent symptoms despite PPI therapy. Aliment Pharmacol Ther. 2015;41:649-61.

55. Chen CL, Yi CH, Liu TT, Orr WC. Effects of mosapride on secondary peristalsis in patients with ineffective esophageal motility. Scand J Gastroenterol. 2013;48:1363-70.

56. Kessing BF, Smout AJ, Bennink RJ, Kraaijpoel N, Oors JM, Bredenoord AJ. Prucalopride decreases esophageal acid exposure and accelerates gastric emptying in healthy subjects. Neurogastroenterol Motil. 2014;26:1079-86.

57. Clayton SB, Patel R, Richter JE. Functional and Anatomic Esophagogastic Junction Outflow Obstruction: Manometry, Timed Barium Esophagram Findings, and Treatment Outcomes. Clin Gastroenterol Hepatol. 2016;14:907-11.

58. Van Hoeij FB, Smout AJ, Bredenoord AJ. Characterization of idiopathic esophagogastric junction outflow obstruction. Neurogastroenterol Motil. 2015;27:1310-6. 
59. Scherer JR, Kwiatek MA, Soper NJ, Pandolfino JE, Kahrilas PJ. Functional esophagogastric junction obstruction with intact peristalsis: a heterogeneous syndrome sometimes akin to achalasia. J Gastrointest Surg. 2009;13:2219-25.

60. Monrroy H, Cisternas D, Bilder C, Ditaranto A, Remes-Troche J, Meixueiro A, Zavala MA, Serra J, Marín I, Ruiz de León A, Pérez de la Serna J, Hani A, Leguizamo A, Abrahao L, Coello R, Valdovinos MA. The Chicago Classification 3.0 Results in More Normal Findings and Fewer Hypotensive Findings With No Difference in Other Diagnoses. Am J Gastroenterol. 2017;112:606-12.

61. Ong AML, Namasivayam V, Wang YT. Evaluation of symptomatic esophagogastric junction outflow obstruction. J Gastroenterol Hepatol. 2018;33:1745-50.

62. Babaei A, Szabo A, Yorio SD, Massey BT. Pressure exposure and catheter impingement affect the recorded pressure in the Manoscan 360 ${ }^{\mathrm{TM}}$ system. Neurogastroenterol Motil. 2018.

63. Krause AJ, Su H, Triggs JR, Beveridge C, Baumann AJ, Donnan E, Pandolfino JE, Carlson DA. Multiple rapid swallows and rapid drink challenge in patients with esophagogastric junction outflow obstruction on high-resolution manometry. Neurogastroenterol Motil. 2021;33:e14000.

64. Patcharatrakul T, Alkaddour A, Pitisuttithum P, Jangsirikul S, Vega KJ, Clarke JO, Gonlachanvit S. How to approach esophagogastric junction outflow obstruction? Ann N Y Acad Sci. 2020;1481:210-23.

65. Schupack D, Katzka DA, Geno DM, Ravi K. The clinical significance of esophagogastric junction outflow obstruction and hypercontractile esophagus in high resolution esophageal manometry. Neurogastroenterol Motil. 2017;29:1-9.

66. Gockel I, Eckardt VF, Schmitt T, Junginger T. Pseudoachalasia: a case series and analysis of the literature. Scand J Gastroenterol. 2005;40:378-85.

67. Lee HR, Lennon VA, Camilleri M, Prather CM. Paraneoplastic gastrointestinal motor dysfunction: clinical and laboratory characteristics. Am J Gastroenterol. 2001;96:373-9.

68. Samo S, Qayed E. Esophagogastric junction outflow obstruction: Where are we now in diagnosis and management? World J Gastroenterol. 2019;25:411-7.

69. Ghisa M, Laserra G, Marabotto E, Ziola S, Tolone S, de Bortoli N, Frazzoni M, Mauro A, Penagini R, Savarino V, Barberio B, Giannini EG, Zentilin P, Gyawali CP, Savarino E. Achalasia and Obstructive Motor Disorders Are Not Uncommon in Patients With Eosinophilic Esophagitis. Clin Gastroenterol Hepatol. 2020.
70. Okeke FC, Raja S, Lynch KL, Dhalla S, Nandwani M, Stein EM, Chander Roland B, Khashab MA, Saxena P, Kumbhari $\mathrm{V}$, Ahuja NK, Clarke JO. What is the clinical significance of esophagogastric junction outflow obstruction? evaluation of 60 patients at a tertiary referral center. Neurogastroenterol Motil. 2017;29.

71. Porter RF, Gyawali CP. Botulinum toxin injection in dysphagia syndromes with preserved esophageal peristalsis and incomplete lower esophageal sphincter relaxation. Neurogastroenterol Motil. 2011;23:139-44, e27-8.

72. Khashab MA, Familiari P, Draganov PV, Aridi HD, Cho JY, Ujiki M, Rio Tinto R, Louis H, Desai PN, Velanovich V, Albéniz E, Haji A, Marks J, Costamagna G, Devière J, Perbtani Y, Hedberg M, Estremera F, Martin Del Campo LA, Yang D, Bukhari M, Brewer O, Sanaei O, Fayad L, Agarwal A, Kumbhari V, Chen YI. Peroral endoscopic myotomy is effective and safe in non-achalasia esophageal motility disorders: an international multicenter study. Endosc Int Open. 2018;6:E1031-6.

73. Schneider HA, Yonker RA, Longley S, Katz P, Mathias J, Panush RS. Scleroderma esophagus: a nonspecific entity. Ann Intern Med. 1984;100:848-50.

74. Ho SC, Chang CS, Wu CY, Chen GH. Ineffective esophageal motility is a primary motility disorder in gastroesophageal reflux disease. Dig Dis Sci. 2002;47:652-6.

75. Su A, Gandhy R, Barlow C, Triadafilopoulos G. Clinical and manometric characteristics of patients with Parkinson's disease and esophageal symptoms. Dis Esophagus. 2017;30:1-6.

76. Roman S, Hirano I, Kwiatek MA, Gonsalves N, Chen J, Kahrilas PJ, Pandolfino JE. Manometric features of eosinophilic esophagitis in esophageal pressure topography. Neurogastroenterol Motil. 2011;23:208-14, e111.

77. Babaei A, Szabo A, Shad S, Massey BT. Chronic daily opioid exposure is associated with dysphagia, esophageal outflow obstruction, and disordered peristalsis. Neurogastroenterol Motil. 2019;31:e13601.

78. Pérez-Fernández MT, Santander C, Marinero A, Burgos-Santamaría D, Chavarría-Herbozo C. Characterization and follow-up of esophagogastric junction outflow obstruction detected by high resolution manometry. Neurogastroenterol Motil. 2016;28:116-26. 